\title{
Star Formation at Zero and Very Low Metallicities
}

\author{
Jonathan C. Tan* and Christopher F. McKee ${ }^{\dagger}$ \\ ${ }^{*}$ Department of Astronomy, University of Florida, Gainesville, FL 32611, USA. \\ jt@astro.ufl.edu \\ ${ }^{\dagger}$ Departments of Physics \& Astronomy, University of California, Berkeley, CA 94720, USA. \\ cmckee@astro.berkeley.edu
}

\begin{abstract}
We describe how star formation is expected to proceed in the early metal-free Universe, focusing on the very first generations of stars. We then discuss how the star formation process may change as the effects of metallicity, external radiative feedback, and magnetic and turbulent support of the gas become more important. The very first stars (Pop III.1) have relatively simple initial conditions set by cosmology and the cooling properties of primordial gas. We describe the evolution of these stars as they grow in mass by accretion from their surrounding gas cores and how the accretion process is affected and eventually terminated by radiative feedback processes, especially $\mathrm{H}$ II region expansion and disk photoevaporation. The ability of the protostar and its disk to generate dynamically important magnetic fields is reviewed and their effects discussed. Pop III.1 star formation is likely to produce massive $\left(\sim 100-200 M_{\odot}\right)$ stars that then influence their surroundings via ionization, stellar winds, and supernovae. These processes heat, ionize and metal-enrich the gas, thus altering the initial conditions for the next generation of star formation. Stars formed from gas that has been altered significantly by radiative and/or mechanical feedback, but not by metal enrichment (Pop III.2) are expected to have significantly smaller masses than Pop III.1 stars because of more efficient cooling from enhanced HD production. Stars formed from gas that is metal-enriched to levels that affect the dynamics of the collapse (the first Pop II stars) are also expected to have relatively low masses. We briefly compare the above star formation scenarios to what is known about present-day star formation.
\end{abstract}

Keywords: stars: formation - early universe - cosmology: theory

PACS: $97.10 . \mathrm{Bt}, 97.20 . \mathrm{Wt}$

\section{INTRODUCTION}

The first generation of stars is expected to have played a significant role in reionizing the Universe, for which observational constraints can be derived from CMB polarization [1] and future high redshift $21 \mathrm{~cm} \mathrm{HI}$ observations [2]. The first stars polluted their pristine surroundings with metals and may have contributed to the observed metal abundances of Galactic halo stars [3] and the Ly- $\alpha$ forest [4, 5]. Light from the first stars may contribute to the observed NIR background intensity, e.g. [6, 7], and its fluctuations [8] (however, see [9]). If massive, the deaths of the first stars may be observable as supernovae [10] or gamma-ray bursts [11]. The first stars thus set the stage for galaxy formation, for which observational constraints now exist at redshifts 8 to 10 [12], and supermassive black hole formation and growth, with $\sim$ billion solar mass black holes being seen out to redshift $6.4[13,14]$.

In discussing the formation of the first stars, the terms "first stars" and "Population III stars" are often used interchangeably, but this can lead to confusion. To be precise, following McKee \& Tan [15], we shall define Population III stars as those stars with a metallicity sufficiently low that it has no effect on either the formation or the evolution of the stars. The value of the critical metallicity for star formation-i.e., the value below which the metals do not influence star formation, e.g. the initial mass function (IMF) - is uncertain, with estimates ranging from $\sim 10^{-6} Z_{\odot}$ if the cooling is dominated by small dust grains that contain a significant fraction of the metals [18] to $\sim 10^{-3.5} Z_{\odot}$ if the cooling is dominated by the fine structure lines of $\mathrm{C}$ and $\mathrm{O}[19]$. It is possible that even lower values of the metallicity could significantly affect the evolution of primordial stars (Meynet, private communication). Among Population III stars, we distinguish between the first and second generations, which we term Population III.1 and III.2, respectively. We define Population III.1 stars as those for which the initial conditions are determined solely by cosmological fluctuations. By definition, the collapse of gas to form these stars is mediated by the cooling properties of primordial or near primordial composition gas that has not been significantly influenced by stellar or AGN radiation fields. Since we expect the effects of radiation to be more pervasive than the spread and mixing of metals, the composition of Pop III.1 halos should in fact be precisely that resulting from big bang nucleosynthesis.

As discussed later in this article, the initial conditions for Population III.2 stars are significantly affected by other stars, primarily by radiation. Lyman-Werner band radiation destroys $\mathrm{H}_{2}$ molecules, reducing the cooling efficiency of the gas. Ionizing radiation heats up the gas to temperatures $\sim 25,000 \mathrm{~K}[16$, 17], depending on the 
temperature of the radiation field. While ionized, thermal expansion can drive the gas out of low-mass dark matter halos. Shocks associated with H II regions and supernova remnants can also ionize and displace the gas. If the gas has a chance to recombine, the relatively high residual electron fraction catalyzes molecule formation, particularly HD formation, which can dramatically enhance subsequent cooling, perhaps reducing the characteristic star-formation mass [20]. Greif \& Bromm[21] use the term "Population II.5" to describe stars that form from gas in which HD cooling is important, whereas in our terminology these would be Population III.2 stars. We believe that it is better to describe all essentially metalfree stars as "Population III." It should be noted that this definition of Population III. 2 stars includes all Population III stars that were significantly affected by previous generations of star formation, even if that did not result in significant HD production. According to Greif \& Bromm [21], Population III.1 stars are relatively rare, constituting only about $10 \%$ by mass of all Population III stars. Note, however, that the precise intensity of the background radiation field that causes a transition from Pop III. 1 to Pop III. 2 remains to be determined from numerical calculations.

\section{POPULATION III.1 STAR FORMATION}

\section{Initial Conditions and Accretion Rate}

The initial conditions for the formation of the first stars are thought to be relatively well understood: they are determined by the growth of small-scale gravitational instabilities from cosmological fluctuations in a cold dark matter universe. The first stars are expected to form at redshifts $z \sim 10-50$ in dark matter "minihalos" of mass $\sim 10^{6} M_{\odot}$ [22]. In the absence of any elements heavier than helium (other than trace amounts of lithium) the chemistry and thermodynamics of the gas are very simple. Once gas collects in the relatively shallow potential wells of the minihalos, cooling is quite weak and is dominated by the ro-vibrational transitions of trace amounts of $\mathrm{H}_{2}$ molecules that cool the gas to $\sim 200 \mathrm{~K}$ at densities $n_{\mathrm{H}} \sim 10^{4} \mathrm{~cm}^{-3}[23,24]$. As the gas core contracts to greater densities, the cooling becomes relatively inefficient and the temperature rises to $\sim 1000 \mathrm{~K}$. At densities $\sim 10^{10} \mathrm{~cm}^{-3}$ rapid 3-body formation of $\mathrm{H}_{2}$ occurs, creating a fully molecular region that can cool much more efficiently. This region starts to collapse supersonically until conditions become optically thick to the line and continuum cooling radiation, which occurs at densities $\sim 10^{17} \mathrm{~cm}^{-3}$. Recent 3D numerical simulations have advanced to densities of order $10^{21} \mathrm{~cm}^{-3}$ (see contributions by Turk et al. and Yoshida et al., these proceedings), but have trouble advancing further given the short simulations timesteps required to resolve the dynamics of the high density gas of the protostar. Further numerical progress can be achieved by introducing sink particles [25].

We have adopted the alternative approach of modeling the protostar's accretion and evolution analytically [26]. The accretion rate depends on the density structure and infall velocity of the gas core at the point when the star starts to form. Omukai \& Nishi [27] and Ripamonti et al. [28] showed that the accreting gas is isentropic with an adiabatic index $\gamma \simeq 1$.1 due to $\mathrm{H}_{2}$ cooling; i.e., each mass element satisfies the relation $P=K \rho^{\gamma}$ with the "entropy parameter" $K=$ const. In hydrostatic equilibrium-and therefore in a subsonic contraction-such a gas has a density profile $\rho \propto r^{-k_{\rho}}$ with $k_{\rho} \simeq 2$.2. This is confirmed by numerical simulations, which show that just before protostar formation the gas at the center of the minihalo has organized itself into an approximately singular polytropic sphere (with a modest degree of flattening about a rotation axis) with $k_{\rho}=2.2$. In our model, we describe the normalization of the density structure of core via the entropy parameter

$$
K^{\prime} \equiv \frac{P / \rho^{\gamma}}{1.88 \times 10^{12} \mathrm{cgs}}=\frac{T_{\mathrm{eff}}^{\prime}}{300 \mathrm{~K}}\left(\frac{10^{4} \mathrm{~cm}^{-3}}{n_{\mathrm{H}}}\right)^{0.1},
$$

where $T_{\text {eff }}^{\prime} \equiv T+\mu \sigma_{\text {turb }}^{2} / k$ is an effective temperature that includes the modest effect of subsonic turbulent motions that are seen in numerical simulations [23].

Simulations show the gas is inflowing subsonically at about a third of the sound speed [23]. We thus use Hunter's [29] solution for mildly subsonic inflow (Mach number $=0.295$ ), with a density that is 1.189 times greater than a singular isothermal sphere at $t=0$, and the accretion rate is 2.6 times greater than the Shu [30] solution.

Feedback from the star, whether due to winds, photoionization, or radiation pressure, can reduce the accretion rate of the star. We define a hypothetical star+disk mass, $m_{* d, 0}$, and accretion rate, $\dot{m}_{* d, 0}$, in the absence of feedback. In this case, the star+disk mass equals the mass of the core out of which it was formed, $m_{* d, 0}=$ $M(r)$. The instantaneous and mean star formation efficiencies are

$$
\begin{gathered}
\varepsilon_{* d} \equiv \frac{\dot{m}_{* d}}{\dot{m}_{* d, 0}}, \\
\bar{\varepsilon}_{* d} \equiv \frac{m_{* d}}{m_{* d, 0}}=\frac{m_{* d}}{M} .
\end{gathered}
$$

Assuming the Hunter solution applies for a singular polytropic sphere with $\gamma=1.1$, the accretion rate is then

$$
\dot{m}_{* d}=0.026 \varepsilon_{* d} K^{\prime 15 / 7}\left(M / M_{\odot}\right)^{-3 / 7} M_{\odot} \mathrm{yr}^{-1},
$$

with the stellar mass smaller than the initial enclosed core mass via $m_{*} \equiv m_{* d} /\left(1+f_{d}\right)=\bar{\varepsilon}_{* d} M /\left(1+f_{d}\right)$. We 


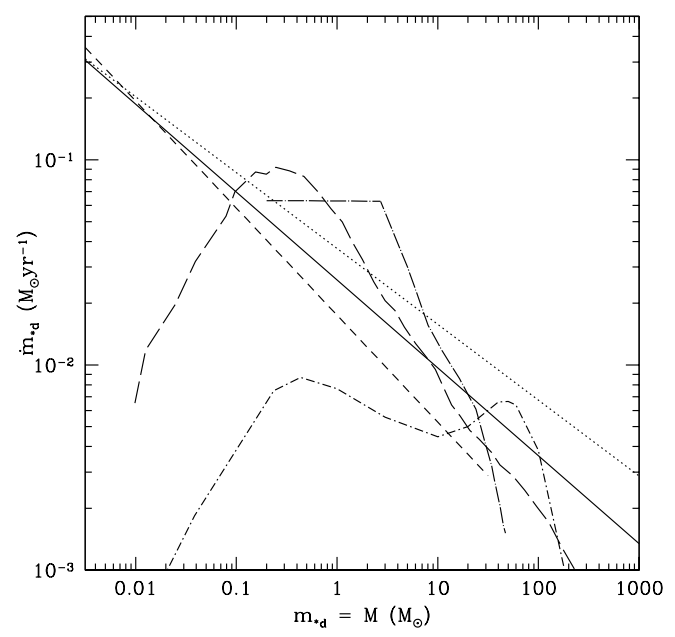

FIGURE 1. Mass accretion rate onto the protostar+disk as a function of their total mass $m_{* d}$ for the case of negligible stellar feedback. Solid line is the fiducial model from Tan \& McKee [26] (with $K^{\prime}=1$ ) from eq. [4]. Dotted line is from the 1D model of Omukai \& Nishi [27]. Dashed line is the analytic result from Ripamonti et al. [28]. Dot-dashed line is the settling inflow rate at the final stage of the simulation of Abel et al. [23], now as a function of the enclosed mass. Note that the decline of this rate at small masses is due to the lack of the full set of high density cooling processes in their simulation. Long-dashed line is the equivalent quantity from Yoshida et al.[31]. Dot-longdashed line is the sink particle accretion rate of Bromm \& Loeb [25].

choose a fiducial value of $f_{d}=1 / 3$ appropriate for disk masses limited by enhanced viscosity due to self-gravity. We compare this analytic accretion rate (for the case of no feedback) with numerical estimates in Fig. 1.

O'Shea \& Norman [32] studied the properties of Pop III.1 pre-stellar cores as a function of redshift. They found that cores at higher redshift are hotter in their outer regions, have higher free electron fractions and so form larger amounts of $\mathrm{H}_{2}\left(\right.$ via H$\left.^{-}\right)$, although these are always small fractions of the total mass. As the centers of the cores contract above the critical density of $10^{4} \mathrm{~cm}^{-3}$, those with higher $\mathrm{H}_{2}$ fractions are able to cool more effectively and thus maintain lower temperatures to the point of protostar formation. The protostar thus accretes from lower-temperature gas and the accretion rates, proportional to $c_{s}^{3} \propto T^{3 / 2}$, are smaller. Measuring infall rates at the time of protostar formation at the scale of $M=100 M_{\odot}$, O'Shea \& Norman find accretion rates of $\sim 10^{-4} M_{\odot} \mathrm{yr}^{-1}$ at $z=30$, rising to $\sim 2 \times 10^{-2} M_{\odot} \mathrm{yr}^{-1}$ at $z=20$. This corresponds to a range in $K^{\prime}$ of 0.20 to 2.4. However, for Hunter's mildly subsonic solution, which we have suggested is closest to the numerical simulations, the accretion rate increases from $0.70 c_{s}^{3} / G$ at large radii $\left(r \gg c_{s} t\right.$, where $t=0$ is the moment of protostar formation) to $2.58 c_{s}^{3} / G$ at small radii (Hunter 1977), an increase of a factor 3.7. This demonstrates that caution should be exercised in inferring accretion rates at late times from those measured at early times.

Spolyar et al. [33] considered the effect of heating due to WIMP dark matter annhilation on the collapse of Pop III.1 pre-stellar cores. In their fiducial model of the dark matter density profile and for a WIMP mass of $100 \mathrm{GeV}$, heating dominates cooling (as evaluated by Yoshida et al. [31]) for $n_{\mathrm{H}}>10^{13} \mathrm{~cm}^{-3}$, i.e. in the inner $\sim 20 \mathrm{AU}$. In this case the collapse can be expected to be halted inside this region and a quasi-hydrostatic object created, although detailed models of the dynamics remain to be worked out. However, as more baryons from the outer regions cool and join this central core, the luminosity needed to support it increases rapidly with mass; the increase provided by WIMP annihilation is uncertain. However, in view of the fact that most of the later accretion of baryons should occur through a disk (see below), which would tend to enhance the baryon to dark matter mass ratio in the central disk and star, we expect any heating due to WIMP annhilation will diminish in importance as the stellar mass grows. It should be noted that the WIMP annhilation heating rate depends sensitively on the density profile of the dark matter, which is so far not adequately resolved in numerical simulations of Pop III.1 core formation.

\section{Protostar and Accretion Disk Evolution}

Assuming dark matter annhilation does not have a significant effect on the main phase of protostellar accretion and noting that there may be a range of accretion rates depending on the formation redshift [32], we now consider what happens to the collapsing gas as it forms a protostar and accretion disk at rates that are initially $\gtrsim 10^{-2} M_{\odot} \mathrm{yr}^{-1}$ (i.e. eq. 4 with $K^{\prime} \sim 1$ ).

Rotation of the infalling gas has a dramatic effect on the evolution of the protostar, since it leads to lower gas densities and optical depths in the regions near the stellar surface that are above and below the disk. This leads smaller photospheric radii and thus higher temperature radiation fields that can then have a stronger dynamical influence on the infall. Following the treatment of Tan \& McKee [26], we parameterize the rotation in terms of

$$
f_{\text {Kep }} \equiv \frac{v_{\text {rot }}\left(r_{\mathrm{sp}}\right)}{v_{\text {Kep }}\left(r_{\mathrm{sp}}\right)},
$$

the ratio of the rotational velocity to the Keplerian velocity measured at the sonic point at $r_{\mathrm{sp}}$. Averaging in spherical shells, Abel, Bryan, \& Norman [23] found $f_{\text {Kep }} \sim 0.5$ independent of radius, so we adopt this as a fiducial value. If angular momentum is conserved inside the sonic 
point, then the accreting gas forms a disk with an outer radius

$$
\begin{aligned}
r_{d} & =1280\left(\frac{f_{\mathrm{Kep}}}{0.5}\right)^{2}\left(\frac{m_{* d, 2}}{\bar{\varepsilon}_{* d}}\right)^{9 / 7} K^{\prime-10 / 7} \mathrm{AU} \\
& \rightarrow 1850\left(\frac{f_{\mathrm{Kep}}}{0.5}\right)^{2} \frac{m_{*, 2}^{9 / 7}}{K^{\prime 10 / 7}} \mathrm{AU}
\end{aligned}
$$

where the $\rightarrow$ is for the case with $f_{d}=1 / 3$.

The first gas to collapse has a small diskcircularization radius and falls directly on to the protostar. The size of the protostar depends on the accretion rate during its formation history. At lower masses there is a balance in the size set by the need to radiate the luminosity, which is mostly due to accretion, with a photospheric temperature that is likely to be close to $\sim 6000 \mathrm{~K}$ because the opacity due to $\mathrm{H}^{-}$rapidly declines below this temperature. Under the assumption of spherical accretion, Stahler, Palla, \& Salpeter [34] found the protostellar radius to be

$$
r_{*} \simeq 67\left(m_{*} / M_{\odot}\right)^{0.27} \dot{m}_{*-2}^{0.41} \quad R_{\odot},
$$

where $\dot{m}_{*,-2} \equiv \dot{m}_{*} /\left(10^{-2} M_{\odot} \mathrm{yr}^{-1}\right)$. For the accretion rates typical of primordial star formation we see that the size is large, but small compared to the disk radius for masses $\gtrsim M_{\odot}$.

As the star grows in mass its internal luminosity increases rapidly and begins to dominate over accretion luminosity [35, 26]. Once the star is older than its instantaneous Kelvin-Helmholz time (i.e. its gravitational energy divided by its internal luminosity), it begins to contract towards the main sequence configuration. At this time there is an associated temporary swelling of the outer layers of star by a factor of about two due to structural rearrangements inside the star. According to Schaerer [36], the main sequence radius for nonrotating stars is

$$
r_{*} \simeq 4.3 m_{*, 2}^{0.55} R_{\odot} \quad \text { (main sequence) }
$$

to within $6 \%$ for $0.4 \leq m_{*, 2} \leq 3$. If the star continues to accrete and gain mass, it will approximately follow this mass-size relation, although rotation will tend to swell the equatorial surface layers somewhat. The evolution of the protostellar radius is shown for several models in Fig. 2

The high accretion rates of primordial protostars make it likely that the disk will build itself up to a mass that is significant compared to the stellar mass. At this point the disk becomes susceptible to global ( $m=1$ mode) gravitational instabilities [38, 39], which are expected to be efficient at driving inflow to the star, thus regulating the disk mass. Thus we assume a fixed ratio of disk to stellar mass, $f_{d}=1 / 3$, in our models.

Accretion through the disk may also be driven by local instabilities, the effects of which can be approximated

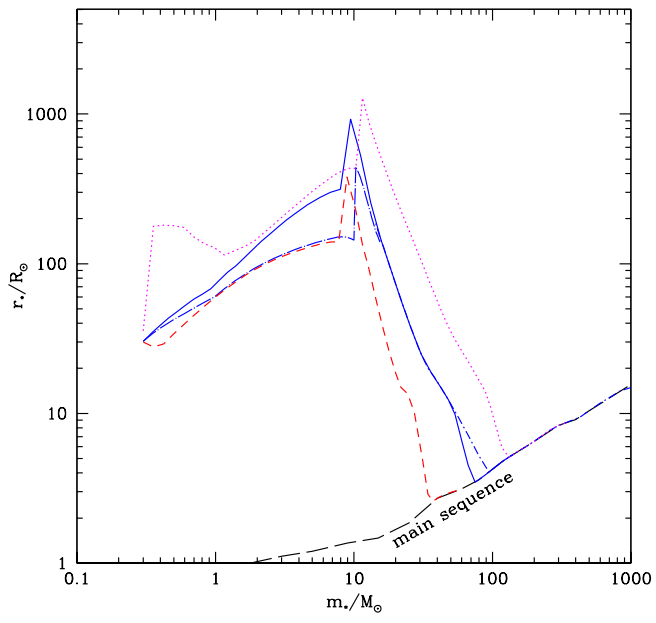

FIGURE 2. Evolution of protostellar radius with mass, based on the model of Tan \& McKee [26]. All models shown have a rotation parameter $f_{\text {Kep }}=0.5$. The solid line shows the evolution of the fiducial model with $K^{\prime}=1$, a Shakura-Sunyaev disk viscosity parameter of $\alpha_{\mathrm{ss}}=0.01$, and a reduction of accretion rate due to feedback effects [15], which becomes important for $m_{*} \gtrsim 50 M_{\odot}$. The star joins the main sequence [36], shown by the long dashed line, at about $80 M_{\odot}$. The dotdashed line, visible only from $50-100 M_{\odot}$, shows the same model but with no reduction in accretion rate due to feedback effects. The dot-long-dashed line, visible up to about $20 M_{\odot}$ shows the fiducial model, but with $\alpha_{\mathrm{ss}}=0.3$ (appropriate for viscosity driven by self-gravity [37]). The dotted line shows the fiducial model but with $K^{\prime}=2$, while the dashed line shows the $K^{\prime}=0.5$ case.

by simple Shakura-Sunyaev $\alpha_{\mathrm{ss}}$-disk models. Two dimensional simulations of clumpy, self-gravitating disks show self-regulation with $\alpha_{\mathrm{ss}} \simeq\left(\Omega t_{\mathrm{th}}\right)^{-1}$ up to a maximum value $\alpha_{\mathrm{ss}} \simeq 0.3$ [37], where $\Omega$ is the orbital angular velocity, $t_{\mathrm{th}} \equiv \Sigma k T_{\mathrm{c}, \mathrm{d}} /\left(\sigma T_{\text {eff, }}^{4}\right)$ is the thermal timescale, $\Sigma$ is the surface density, $T_{\mathrm{c}, \mathrm{d}}$ is the disk's central (midplane) temperature, and $T_{\text {eff, }}$ the effective photospheric temperature at the disk's surface. Fragmentation occurs when $\Omega t_{\text {th }} \lesssim 3$ : this condition has the best chance of being satisfied in the outermost parts of the disk that are still optically thick. However, Tan \& Blackman [40] considered the gravitational stability of constant $\alpha_{\mathrm{ss}}=0.3$ disks fed at accretion rates given by eq. 4 and found that the optically thick parts of the disk remained Toomre stable $(Q>1)$ during all stages of the growth of the protostar. We therefore expect that most Pop III.1 accretion disks, at least in their early stages, will grow in mass and mass surface density to the point at which gravitational instabilities, both global and local, then mediate accretion to the star.

In addition to gravitational instabilities, the magnetorotational instability (MRI) may develop if dynamically 
important magnetic fields are generated by dynamo action in the disk (see below). This process could be particularly important in the inner accretion disk, where gravitational instability is suppressed. The MRI is thought to yield viscous stresses that correspond to somewhat smaller values of $\alpha_{\mathrm{ss}} \sim 0.01$ [41], although the actual effective value remains unclear.

\section{Generation of Magnetic Fields in the Accretion Disk}

Given the right conditions, dynamo action can amplify a seed magnetic field. The basic requirement is that the field is frozen into the gas and that gas motions repeatedly stretch the field, e.g. via turbulent or shearing motions. Here we briefly describe the results of Tan \& Blackman [40], who considered these issues.

The seed field for the primordial star-forming environment can be estimated from the Biermann battery mechanism for non-barotropic flows. On scales much larger than that of an individual star, numerical simulations indicate that by $z=18$, seed fields of order $\bar{B}_{0} \sim 10^{-26}$ Gauss can be generated [42]. We assume such a field is generated inside the region around the minihalo on scales $\sim 100 \mathrm{pc}$. The infall speeds within this region are of order $1 \mathrm{~km} \mathrm{~s}^{-1}$, vastly greater than the drift speeds of the ions through the neutrals (the ionization fractions are much greater than the equilibrium values for the gas, which has a minimum temperature of $\sim 200 \mathrm{~K}$ ). Thus the field is frozen into the contracting gas: going from parsec to $\mathrm{AU}$ scales increases the field strength by a factor of $\sim 10^{10}$, so the seed field in the disk may then be $\sim 10^{-16} \mathrm{G}$. This estimate is highly uncertain. Seed fields several orders of magnitude larger could arise in the protostellar disk from the Biermann battery term if the pressure and density gradients are misaligned inside this region as well. A further complication that could lead to lower field strengths is the possibility of efficient turbulent diffusion of the field so that flux freezing and field advection do not play as significant a role. However, since the disk-dynamo amplifies the initial field exponentially, the uncertainties in the strength of the seed field do not significantly affect the time to reach saturation.

A seed field is expected to be amplified by a combination of shearing and turbulent motions in the disk. As discussed above, initially the turbulence is thought to be due to self-gravity [37], but as the field strength grows, this could be replaced by the MRI. The field strength grows exponentially with a growth time $\sim \alpha_{\mathrm{ss}}^{-1 / 2} \Omega^{-1}$. Field growth stops when the fields become dynamically important, i.e. for $B \sim(4 \pi \rho)^{1 / 2} \alpha_{\mathrm{ss}} c_{s}$. The generation of strong B-fields that are ordered on scales large compared to the disk thickness requires turbulence in a stratified disk: the helical disk dynamo[43]. More specifically the turbulence must generate net helicity in each hemisphere. If this occurs, and numerical simulations are required for confirmation, then these large-scale B-fields could start to drive a hydromagnetic bipolar disk wind, whose feedback effects will be discussed below.

The presence of strong B-fields in the disk and their possible advection into the protostar, then allows a stardisk interaction that can help the star to shed angular momentum and may set the rotation rate with which it is born. Note that models of primordial protostellar evolution predict that the star is mostly radiatively stable in the pre-H nuclear burning phase, which implies there is very little dynamo amplification of B-field in the star independent of the disk.

\section{Radial Structure of the Accretion Disk}

Given these considerations, a first approximation for the radial structure of the protostellar accretion disk can be made by using the standard theory of steady, thin, viscous accretion disks, with a spatially constant viscosity parameter, $\alpha_{\mathrm{ss}}$, and for simplicity ignoring energy injection from the star. Tan \& McKee [26] calculated the evolution of the radial structure, assuming the disk is fed at a rate given by equation (4) and the inner boundary is at the protostellar surface, $r_{*}$. The viscosity is assumed to be a function of the total pressure. Typically gas pressure dominates over radiation pressure in the earlier stages $\left(m_{*} \lesssim 20 M_{\odot}\right)$. We chose $\alpha_{\text {ss }}=0.01$ as a fiducial value, that may arise from viscosity provided by the magneto rotational instability [41].

The radial structure of the disk is governed by the equations of energy conservation and of angular momentum conservation. Energy conservation gives the emergent flux as

$$
\begin{aligned}
F_{0} & =\frac{\dot{m}_{*}}{4 \pi \varpi} \frac{\partial}{\partial \varpi}\left(\frac{5}{3} \bar{\varepsilon}_{\mathrm{th}}+\bar{\varepsilon}_{I}\right)+\frac{3 G m_{*} \dot{m}_{*} f}{8 \pi \bar{\varpi}^{3}} \\
& \equiv \phi_{I}\left(\frac{3 G m_{*} \dot{m}_{*} f}{8 \pi \bar{\varpi}^{3}}\right)
\end{aligned}
$$

Here

$$
f \equiv 1-\left(\frac{\varpi_{0}}{\varpi}\right)^{1 / 2},
$$

is the factor that embodies the boundary condition that angular momentum cannot be transferred across a surface on which the angular velocity has no gradient; $\varpi_{0}$ is the cylindrical radius at which $\partial \Omega / \partial \varpi$ vanishes, which we take to be equal to the stellar radius. The dimensionless factor $\phi_{I}$ describes the advection of thermal and internal energy in the disk and is generally less than unity.

To evaluate the angular momentum transfer in the disk, we adopt the $\alpha$-disk model of Shakura \& Sunyaev [44], 
in which the transverse stress in the disk is proportional to the pressure, $w_{\varpi \phi}=-\frac{3}{2} \alpha P$ (we have included the factor $\frac{3}{2}$ to conform with the convention of Frank et al. [45]). The equation describing angular momentum transport is then

$$
\dot{m}_{*} \Omega f=6 \pi \alpha \int_{0}^{z_{s}} P d z
$$

where $z_{s}$ is the height of the surface of the disk.

The radial disk structure at three different stages $\left(m_{*}=\right.$ $\left.1,10,100 M_{\odot}\right)$ is shown in Fig. 3, with the radial coordinate measured in units of the protostellar radius.

\section{Vertical Structure of the Accretion Disk}

McKee \& Tan [15] calculated the vertical structure of Pop III.1 accretion disks. The vertical structure of the disk is governed by three equations: first is the first moment of the radiative transfer equation,

$$
\frac{\partial P_{\mathrm{rad}}}{\partial z}=-\frac{\rho \kappa_{F} F}{c},
$$

where $\kappa_{F}$ is the flux-weighted mean opacity per unit mass and $F(z)$ is the radiative flux. We assume that the effective optical depth for true absorption, $\tau^{*}=$ $\left(\tau_{\text {abs }} \tau_{\text {scatt }}\right)^{1 / 2}$, is much greater than unity so that the gas is approximately in LTE [44, 46]. Then $P_{\text {rad }} \simeq \frac{1}{3} a T^{4}$ and $\kappa_{F} \simeq \kappa_{\mathrm{R}}$, where $\kappa_{\mathrm{R}}$ is the Rosseland mean opacity per unit mass, so that equation (13) reduces to the equation of radiative diffusion,

$$
\frac{\partial T}{\partial z}=-\frac{3 \kappa_{\mathrm{R}} \rho F}{16 \sigma T^{3}} .
$$

The second equation describes the growth of the flux due to viscous dissipation [47],

$$
\frac{\partial F}{\partial z}=-\phi_{I} w_{\varpi \phi} \varpi \frac{\partial \Omega}{\partial \varpi}=\frac{9}{4} \phi_{I} \alpha \Omega P .
$$

We have included the factor $\phi_{I}$ to allow for the reduction in the flux by the advection of internal energy. In addition to the factor $\frac{3}{2} \phi_{I}$, equation (15) differs from the expression adopted by Shakura \& Sunyaev [44] in that it has $\partial F / \partial z \propto P$ rather than $\propto \rho$. One can show, however, that the height of the disk is very insensitive to this change. Integration of equation (15) together with equation (12) leads directly to the energy equation (10).

Finally, we have the equation of hydrostatic equilibrium,

$$
\frac{\partial P}{\partial z}=-\frac{\rho G m_{*} z}{\varpi^{3}}
$$

where the pressure $P$ includes both gas pressure and radiation pressure,

$$
P=P_{g}+P_{\mathrm{rad}}=\frac{\rho k T}{\mu}+\frac{4 \sigma T^{4}}{3 c} .
$$

A numerical solution of these equations using the opacities of Iglesias \& Rogers [48] is shown in Fig. 4 for one particular stage of the protostellar evolution for a location at $10 R_{*}$ around a $100 M_{\odot}$ main sequence star, accreting at $2.4 \times 10^{-3} M_{\odot} \mathrm{yr}^{-1}$, i.e. the fiducial rate from a $K^{\prime}=1$ core with no reduction due to feedback.

More generally, we follow the disk structure during the course of the protostellar evolution (i.e. as $m_{*}, \dot{m}_{*}$ and $r_{*}$ evolve). As we discuss below, the disk thickness is one important factor in determining the stellar mass at which accretion is shut off. At masses $m_{*} \sim 100 M_{\odot}$ we find the aspect ratio of the disk surface, $z_{s} / r$, is about twice $h / r$, where $h$ is the scale height, and has a very weak dependence on radius.

\section{Feedback Processes and the IMF}

\section{Radiative Feedback}

The evolution of the bolometric luminosity of the protostar and the inner accretion disk is shown in Fig. 5 for three different accretion rates corresponding to $K^{\prime}=$ $0.5,1,2$. The luminosities are sub-Eddington for almost the entire evolution. Only the $K^{\prime}=2$ model (with the highest accretion rate) exceeds the Eddington limit: at the low-mass limit this is due to the initial condition having a stellar radius that is too small and the star quickly adjusts by undergoing rapid expansion; at about $80 M_{\odot}$ the star is close to joining the main sequence and so has a relatively small size and is still accreting relatively efficiently because its H II region is still confined (see below), thus resulting in a high accretion luminosity. However, in this latter case we do not expect accretion to be significantly affected by radiation pressure because much of the luminosity will be directed in polar directions and most of the accretion is of neutral gas from the equatorial regions.

As discussed in McKee \& Tan [15], we expect ionizing feedback to be more important than continuum radiation pressure at reducing accretion efficiency and eventually setting the final stellar mass. The $\mathrm{H}$-ionizing photon luminosities for the $K^{\prime}=0.5,1,2$ models are shown in Fig. 6. At lower masses, the protostars are much larger than the corresponding main sequence stars and so have cooler photospheric temperatures and smaller ionizing luminosities. Once the protostar is older than about its instantaneous Kelvin-Helmholz time, which occurs at higher masses for higher accretion rates, the star contracts towards the main sequence and the ionizing luminosity rises accordingly.

Figure 7 gives an overview of the ionizing feedback processes occurring near the protostar. At early stages the ionizing flux from the protostar is smaller than the flux of accreting neutral atoms to its surface and the H II 


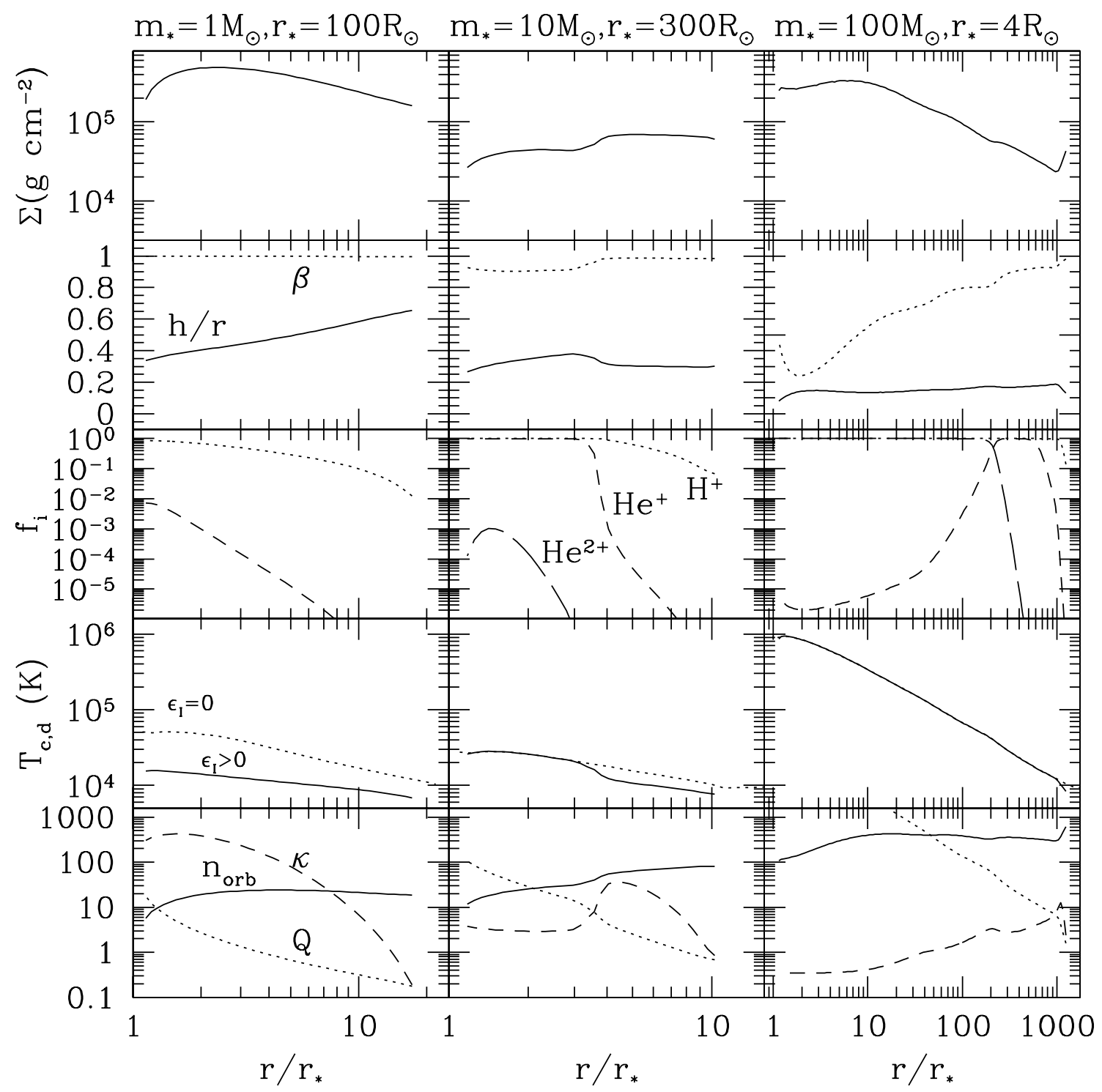

FIGURE 3. Protostellar disk structure [40] for models with $\alpha_{\mathrm{ss}}=0.01$ and $m_{*}=1,10,100 M_{\odot}$, for which $r_{*}=100,300,4 R_{\odot}$ and $\dot{m}_{*}=(17,6.4,2.4) \times 10^{-3} M_{\odot} \mathrm{yr}^{-1}$, respectively, i.e. from eq. 4 with no feedback. From top to bottom the panels show (1) surface density, $\Sigma$; (2) ratio of scaleheight to radius, $h / r$, and ratio of gas pressure to total pressure, $\beta$; (3) midplane ionization fractions of $\mathrm{H}^{+}, \mathrm{He}^{+}, \mathrm{He}^{2+}$; (4) disk midplane temperature, $T_{\mathrm{c}, \mathrm{d}}$ (the dotted lines show results for when the ionization energy is neglected); (5) number of orbits before accretion to the star, $n_{\text {orb }}$, Toomre $Q$ stability parameter, and Rosseland mean opacity $\kappa$, evaluated at the midplane. Note that all quantities are azimuthal and temporal averages of the disk, which, being turbulent, would exhibit local fluctuations. 


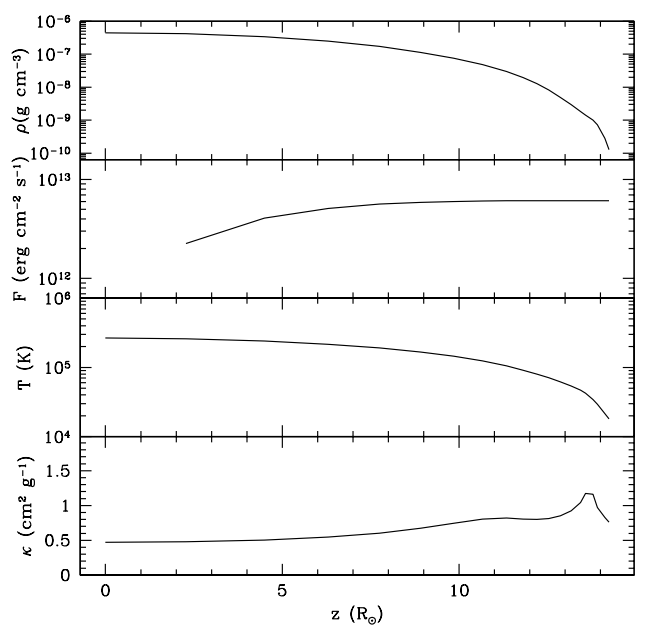

FIGURE 4. Vertical structure of the accretion disk at $r=$ $10 r_{*} \simeq 43 R_{\odot}$ for $m_{*}=100 M_{\odot}, K^{\prime}=1, f_{\text {Kep }}=0.5$ and no reduction in accretion efficiency due to feedback.

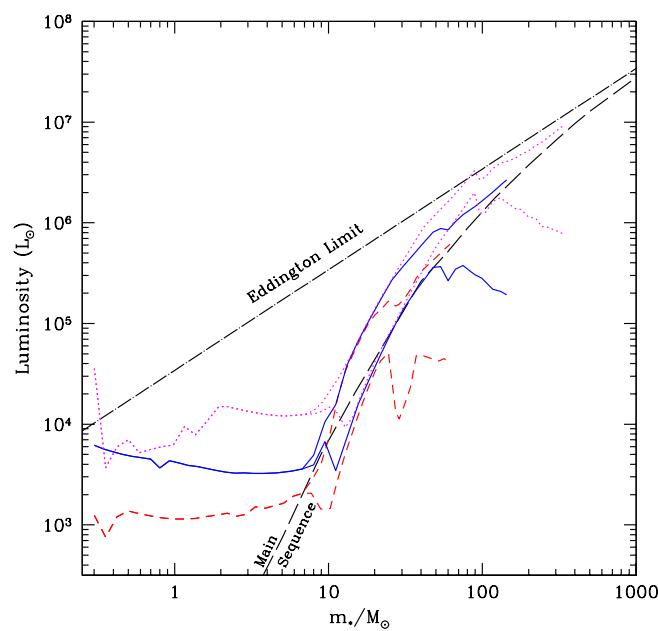

FIGURE 5. Protostellar bolometric luminosities for models with $K^{\prime}=0.5,1,2$ (dashed, solid, dotted lines). In each case the total luminosity is shown with the higher line and the accretion luminosity (boundary layer + inner disk) is shown with the lower line. The Eddington limit is indicated with the dot-longdashed line and the zero age main sequence luminosity [36] with the long-dashed line.

region is confined there. As the ionizing luminosity increases, the $\mathrm{H}$ II region can begin to expand into the infalling envelope, penetrating furthest along the rotation axes. At the ionized-neutral boundary, radiation pressure feedback is exerted due to resonant scattering of FUV radiation in the Lyman- $\alpha$ damping wings. As a result of the high column densities of neutral gas around the $\mathrm{H}$ II region, this radiation is trapped and the pressure am-

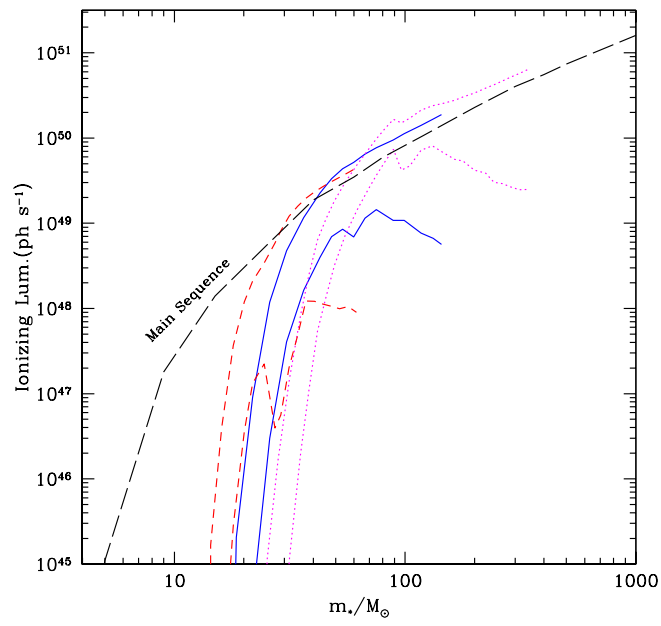

FIGURE 6. Protostellar $\mathrm{H}$-ionizing photon luminosities for models with $K^{\prime}=0.5,1,2$ (dashed, solid, dotted lines). The total luminosity is shown with the higher line and the accretion luminosity (boundary layer + inner disk) is shown with the lower line. The zero age main sequence $\mathrm{H}$-ionizing luminosities [36] are indicated with the long-dashed line.

plified by large factors. For typical rotation parameters $f_{\text {Kep }} \simeq 0.5$, this radiation pressure becomes larger than the ram pressure of the infalling gas in the polar directions for stellar masses of order $20 M_{\odot}$. However, once the infall is reversed at the poles, the Lyman- $\alpha$ photons can escape and the accretion in other directions proceeds relatively unimpeded. Thus we expect Lyman- $\alpha$ radiation pressure to have only a minor effect on the accretion efficiency.

The next feedback effect to occur is the expansion of the $\mathrm{H}$ II region to distances larger than the gravitational escape radius $r_{g}$ for ionized gas. This distance is

$$
r_{g} \equiv \frac{G \phi_{\mathrm{Edd}} m_{* d}}{c_{i}^{2}}=260 \phi_{\mathrm{Edd}}\left(\frac{2.5}{T_{4}}\right) m_{* d, 2} \mathrm{AU}
$$

where $T_{4}$ is the ionized gas temperature in units of $10^{4} \mathrm{~K}$ and we have taken the gravitating mass to be $m_{* d}$ and we have allowed for the decrease in the radius due to radiation pressure from electron scattering through the factor

$$
\phi_{\text {Edd }} \equiv 1-\frac{L}{L_{\text {Edd }}},
$$

where $L_{\mathrm{Edd}}=4 \pi \mathrm{Gmc} / \kappa_{\text {Thomson }}$ is the Eddington limit.

Shortly after the H II region reaches $r_{g}$, pressure forces in the ionized gas become large enough to reverse the infall to the protostar. This effect will occur first in the polar directions, typically at about $50 M_{\odot}$ in our fiducial model. For rotation parameters $f_{\text {Kep }} \simeq 0.5$, expansion in the equatorial directions (just above the accretion disk) 

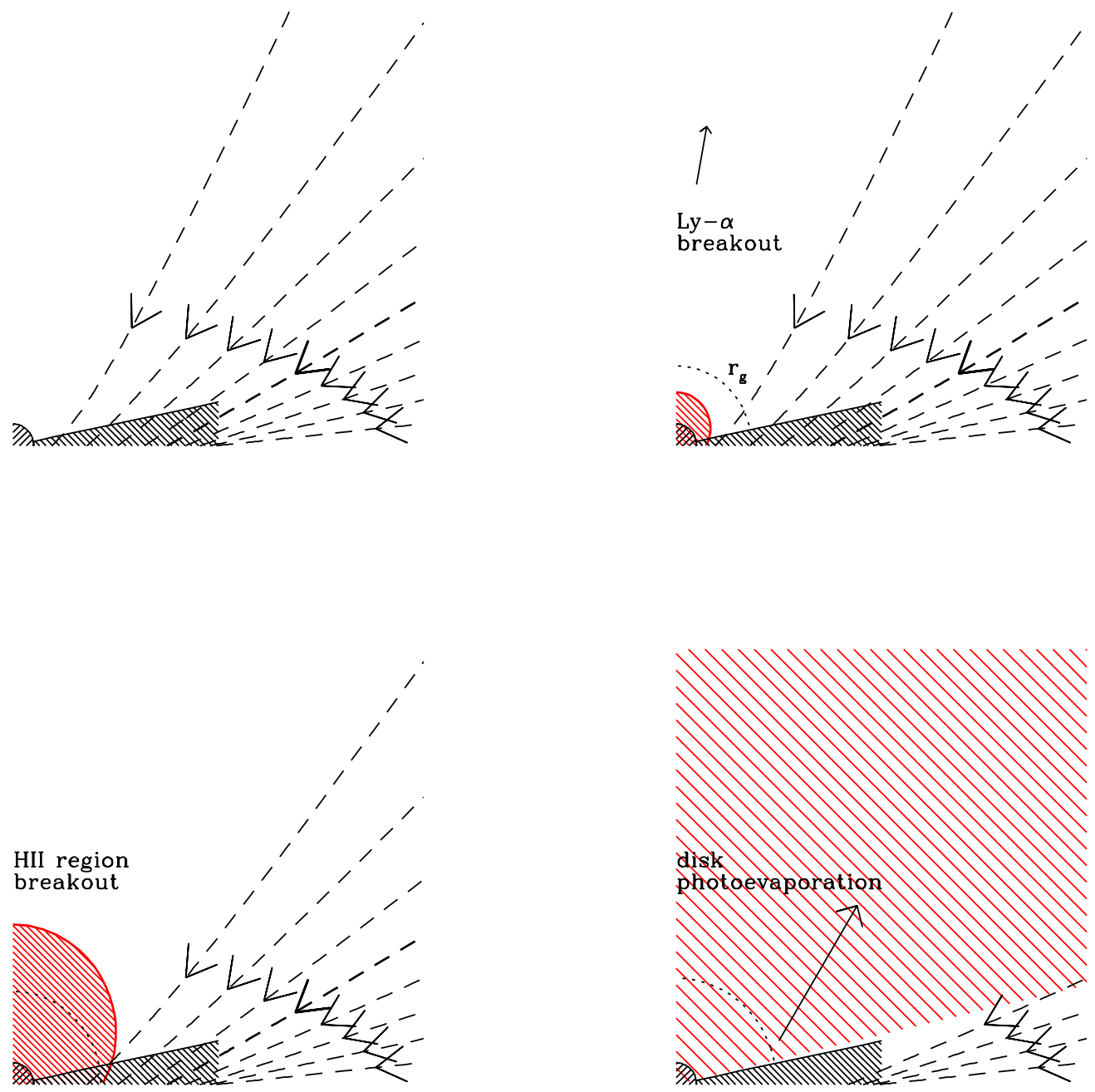

FIGURE 7. Overview of the accretion geometry and feedback processes involved in primordial star formation. (a) Top left: Cross section of the accretion geometry: the dashed lines show streamlines of the rotating, infalling gas, with figure of revolution from each streamline separating $10 \%$ of the total infall from this hemisphere. The aspect ratio of the accretion disk is realistic, while the size of the star has been exaggerated for clarity. (b) Top right: The red shaded region shows the extent of the H II region, which at this relatively early stage is still confined inside the gravitational radius for the escape of ionized gas, $r_{g}$. Lyman- $\alpha$ radiation pressure feedback should be strong enough to prevent accretion in the polar directions. (c) Bottom left: The stellar mass and ionizing luminosity have grown, and the H II region is just in the process of breaking out of the accretion flow. Once a significant volume beyond $r_{g}$ is ionized, accretion from these directions is expected to be shut off. (d) Bottom right: Final stage of accretion involves shadowing of the equatorial region by the disk, which at the same time is photoevaporated. The competition between this photoevaporative outflow and the residual accretion rate sets the final mass of the star. 


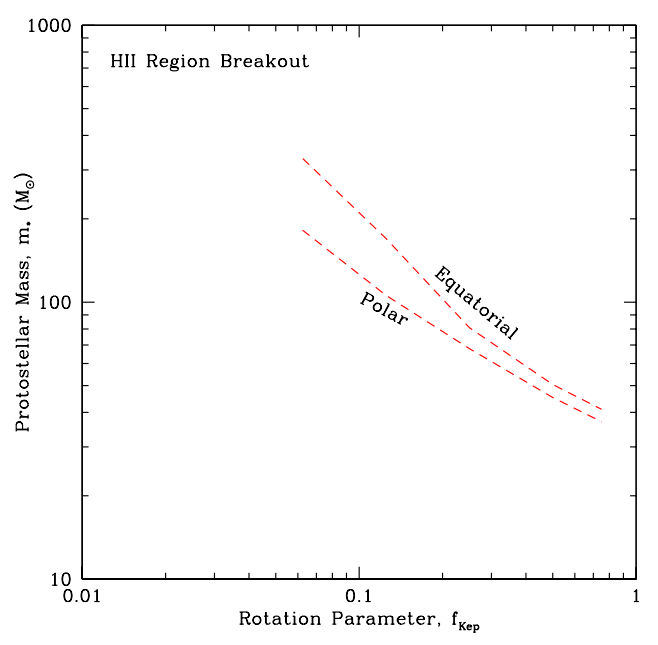

FIGURE 8. Mass scales of H II region break out as a function of the rotation parameter $f_{\text {Kep }}$. The lower dashed line marked "Polar" shows the mass scale of the protostar at which the H II region reaches $r_{g}$ (based on star plus disk mass) along the rotation axis of the protostar. The upper dashed line marked "Equatorial" shows the mass scale of the protostar when the $\mathrm{H}$ II region reaches $r_{g}$ in a direction just above the disk plane $(0.9 \pi / 2$ from the rotation axis).

occurs very soon after polar breakout (see Fig. 8 and Table 1). Higher accretion rates and smaller rotation parameters result in larger mass scales for $\mathrm{H}$ II region breakout.

The protostar and accretion envelope should evolve to a state in which the entire region above and below the accretion disk has been ionized (Fig. $7 \mathrm{f}$ ). No accretion will occur from these directions. The accretion disk, which is still mostly neutral in its outer regions, has a finite thickness and will shield an extended equatorial region from direct ionizing flux from the protostar, allowing accretion to be able to continue from these regions. The reduction in accretion rate caused by the expansion of the H II region and the fraction allowed in the disk-shadowed region is shown in Fig. 9, where the fiducial no-feedback case is also shown.

Also shown in Fig. 9 is the mass loss rate due to the process of disk photoevaporation [49]. Ionization from the protostar creates an ionized atmosphere above the neutral accretion disk, which then scatters some ionizing photons down on to the shielded region of the outer disk, beyond $r_{g}$. An ionized outflow is driven from these regions at a rate

$$
\dot{m}_{\text {evap }} \simeq 4.1 \times 10^{-5} S_{49}^{1 / 2} T_{4}^{0.4} m_{* d, 2}^{1 / 2} M_{\odot} \mathrm{yr}^{-1},
$$

where $S_{49}$ is the H-ionizing photon luminosity in units of $10^{49}$ photons s $^{-1}$. When this rate becomes comparable to the accretion rate to the disk, then we expect further

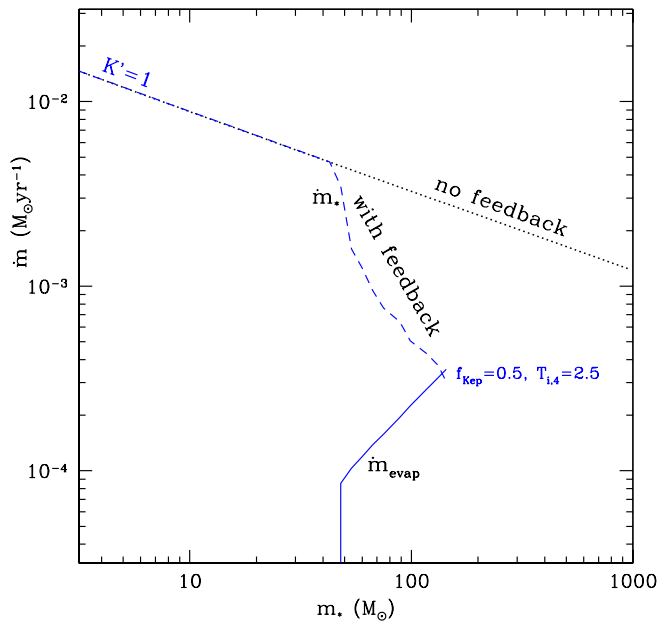

FIGURE 9. Feedback-limited accretion: fiducial case. The evolution of the accretion rate versus protostellar mass is shown for the fiducial model $\left(f_{\mathrm{Kep}}=0.5, K^{\prime}=1, T_{i}=25,000 \mathrm{~K}\right)$ in the cases of "no feedback" and "with feedback". In the latter, the accretion efficiency is reduced as the H II region expands to $r_{g}$ and beyond. However, accretion is allowed to continue from directions that are shadowed by the disk photosphere. The disk structure and protostellar structure and feedback are calculated self-consistently given the evolution in $\dot{m}_{*}$. Also shown is the photoevaporative mass loss rate, $\dot{m}_{\text {evap }}$, which starts once the $\mathrm{H} \mathrm{II} \mathrm{region} \mathrm{has} \mathrm{broken} \mathrm{out} \mathrm{in} \mathrm{the} \mathrm{equatorial} \mathrm{direction} \mathrm{and} \mathrm{grows}$ as the ionizing flux increases. We see that this mass loss rate becomes greater than the accretion rate at $m_{*} \simeq 137 M_{\odot}$, and we identify this mass scale as our best estimate of initial mass scale of the first stars.

growth of the stellar mass to be very limited. For simplicity we equate the final stellar mass to that mass at which the photoevaporative mass loss rate equals the accretion rate. It is about $140 M_{\odot}$ in the fiducial case, and Table 1 and Fig. 10 summarize other cases.

An analytic estimate can be made for the final protostellar mass. Assuming the H-ionizing photon luminosity is mostly due to the main sequence luminosity of the star we have

$$
S \simeq 7.9 \times 10^{49} \phi_{S} m_{*, 2}^{1.5} \quad \mathrm{ph} \mathrm{s}^{-1},
$$

which for $\phi_{S}=1$ is a fit to Schaerer's (2002) results for the ionizing luminosity of main sequence primordial stars; the fit is accurate to within about $5 \%$ for $60 M_{\odot} \lesssim$ $m_{*} \lesssim 300 M_{\odot}$. Then the photoevaporation rate becomes

$$
\begin{aligned}
\dot{m}_{\text {evap }} & =1.70 \times 10^{-4} \phi_{S}^{1 / 2}\left(1+f_{d}\right)^{1 / 2} \\
& \times\left(\frac{T_{4}}{2.5}\right)^{0.4} m_{*, 2}^{5 / 4} M_{\odot} \mathrm{yr}^{-1}
\end{aligned}
$$

The accretion rate onto the star-disk system is given by equation (4). Equating this with equation (22), we find 
TABLE 1. Mass Scales of Population III.1 Protostellar Feedback

\begin{tabular}{|c|c|c|c|c|c|}
\hline$K^{\prime}$ & $f_{\mathrm{Kep}}$ & $T_{i} /\left(10^{4} \mathrm{~K}\right)$ & $m_{*, \mathrm{pb}}\left(M_{\odot}\right)^{*}$ & $m_{*, \mathrm{eb}}\left(M_{\odot}\right)^{\dagger}$ & $m_{*, \text { evap }}\left(M_{\odot}\right)^{* *}$ \\
\hline 1 & 0.5 & 2.5 & 45.3 & 50.4 & $137^{\ddagger}$ \\
\hline 1 & 0.75 & 2.5 & 37 & 41 & 137 \\
\hline 1 & 0.25 & 2.5 & 68 & 81 & 143 \\
\hline 1 & 0.125 & 2.5 & 106 & 170 & 173 \\
\hline 1 & 0.0626 & 2.5 & 182 & $330^{\S}$ & 256 \\
\hline 1 & 0.5 & 5.0 & 35 & 38 & 120 \\
\hline 1 & 0.25 & 5.0 & 53.0 & 61 & 125 \\
\hline 0.5 & 0.5 & 2.5 & 23.0 & 24.5 & 57 \\
\hline 2.0 & 0.5 & 2.5 & 85 & 87 & 321 \\
\hline \multicolumn{6}{|c|}{$\begin{array}{l}{ }^{*} \text { Mass scale of HII region polar breakout. } \\
\text { Mass scale of HII region near-equatorial breakout. } \\
*_{* *} \text { Mass scale of disk photoevaporation limited accretion. } \\
\text { Fiducial model. } \\
\text { This mass is greater than } m_{*, \text { evap }} \text { in this case because it is calculated without allowing } \\
\text { for a reduction in } m_{*} \text { during the evolution due to polar HII region breakout. }\end{array}$} \\
\hline
\end{tabular}

that the resulting maximum stellar mass is

$\operatorname{Max} m_{* f, 2}=6.3 \frac{\varepsilon_{* d}^{28 / 47} \bar{\varepsilon}_{* d}^{12 / 47} \phi_{S}^{14 / 47} K^{160 / 47}}{\left(1+f_{d}\right)^{26 / 47}}\left(\frac{2.5}{T_{4}}\right)^{0.24}$.

Recall that $\varepsilon_{* d}$ is the instantaneous star formation efficiency-i.e., the ratio of the accretion rate onto the star to the rate that would have occurred in the absence of feedback. Here this ratio is just the shadowing factor, $f_{\text {sh }}$, i.e. the fraction of the sky as seen from the protostar that is blocked by the disk. For this simple analytic estimate, we make the approximation that the shadowing sets in when the stellar mass reaches $m_{1}$, so that $\varepsilon_{* d}=1$ until the mass of the central star reaches $m_{1}$ and $\varepsilon_{* d}=f_{\text {sh }}$ thereafter. It is then straightforward to show that

$$
\bar{\varepsilon}_{* d}=\frac{f_{\mathrm{sh}}}{1-\left(1-f_{\mathrm{sh}}\right)\left(m_{1} / m_{* d}\right)},
$$

provided that $m_{* d} \geq m_{1}$. Note that the average efficiency $\bar{\varepsilon}_{* d}=1$ at the onset of shadowing $\left(m_{* d}=m_{1}\right)$ and that $\bar{\varepsilon}_{* d} \rightarrow f_{\text {sh }}$ at late times $m_{* d} \gg m_{1}$. Normalizing $f_{\text {sh }}$ to a typical value of 0.2 we find

$$
\begin{aligned}
\operatorname{Max} m_{* f, 2} & =1.45 K^{160 / 47}\left(\frac{2.5}{T_{4}}\right)^{0.24} \\
& \times\left(\frac{f_{\text {sh }}}{0.2}\right)^{28 / 47}\left(\frac{\bar{\varepsilon}_{* d}}{0.25}\right)^{12 / 47},
\end{aligned}
$$

where we have set the ionizing luminosity factor $\phi_{S}=1$ and the disk mass fraction $f_{d}=\frac{1}{3}$; we have normalized $\bar{\varepsilon}_{* d}$ to a value of 0.25 , which is approximately correct for $K^{\prime}=1$ and for $m_{1} \simeq 50 M_{\odot}$ and $m_{* d}=200 M_{\odot}$. This analytic estimate therefore also suggests that for the fiducial case $\left(K^{\prime}=1\right)$ the mass of a Pop III.1 star should be $\simeq 140 M_{\odot}$.
The uncertainties in these mass estimates include: (1) the assumption that the gas distribution far from the star is approximately spherical - in reality it is likely to be flattened towards the equatorial plane, thus increasing the fraction of gas that is shadowed by the disk and raising the final protostellar mass; (2) uncertainties in the disk photoevaporation mass loss rate due to corrections to the Hollenbach et al. [49] rate from the flow starting inside $r_{g}$ and from radiation pressure corrections; (3) uncertainties in the $\mathrm{H}$ II region breakout mass due to hydrodynamic instabilities and 3D geometry effects; (4) uncertainties in the accretion rate at late times, where self-similarity may break down [25]; (5) the effect of rotation on protostellar models, which will lead to cooler equatorial surface temperatures and thus a reduced ionizing flux in the direction of the disk; (6) the simplified condition, $\dot{m}_{\text {evap }}>\dot{m}_{* d}$, used to mark the end of accretion; and (7) the possible effect of protostellar outflows (discussed below).

\section{Mechanical Feedback}

Protostellar outflows, thought to be launched by largescale magnetic fields threading the inner accretion disk, are ubiquitous from present-day protostars. The momentum flux in these flows typically exceeds that due to radiation pressure by several orders of magnitude. As described above, Tan \& Blackman [40] discussed how turbulence in a stratified disk that generates helicity is the necessary condition for production of dynamicallystrong large-scale magnetic fields by dynamo action. They then considered the effect of such an outflow on the surrounding minihalo gas, following the analysis of Matzner \& McKee [50]. The force distribution of centrifugally-launched hydromagnetic outflows is colli- 


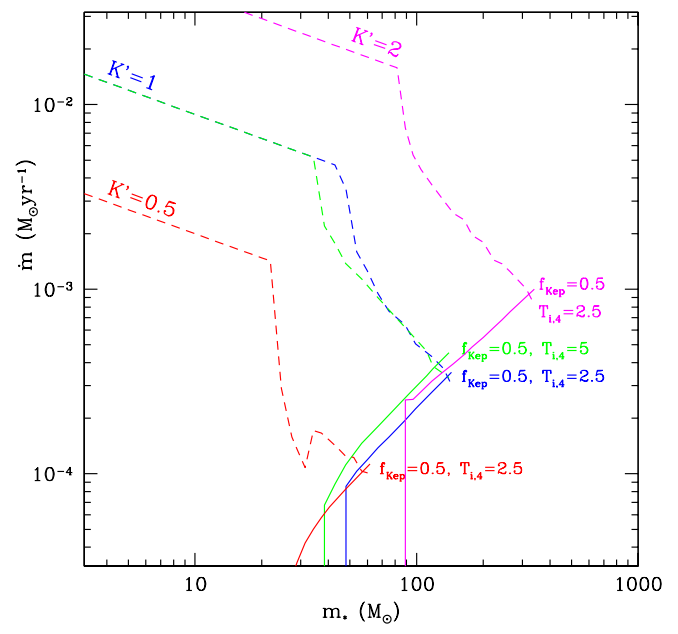

FIGURE 10. Feedback-limited accretion: effect of ionized gas temperature and accretion rate. The fiducial model $\left(f_{\mathrm{Kep}}=\right.$ $0.5, K^{\prime}=1, T_{i, 4}=2.5 \mathrm{~K}$ ) shown in Fig. 9 is compared to models in which one parameter has been changed: a model with $T_{i, 4}=5$ and two models with $K^{\prime}=0.5,2$. The dashed lines show the accretion rate to the star, $\dot{m}_{*}$, and the solid lines show the photoevaporative mass loss rate, $\dot{m}_{\text {evap }}$. The change in temperature causes relatively minor differences, while the change in $K^{\prime}$, equivalent to a change in $\dot{m}_{*}$ of factors of 4.4 above and below the fiducial level, leads to roughly a factor of 2.4 change in the final stellar mass. Note the increase in $\dot{m}_{*}$ for the $K^{\prime}=0.5$ case at around $35 M_{\odot}$ is due to a thickening of the inner accretion disk as the star contracts down to its main sequence configuration and assumes material at large distances still remains to be accreted in the enlarged shadowed region.

mated along the rotation axes, but includes a significant wider-angled component. Matzner \& McKee [51] showed that far from the star the angular distribution of the momentum in a radial hydromagnetic wind can be approximated by

$$
\frac{d p_{w}}{d \Omega}=\frac{p_{w}}{4 \pi} \frac{1}{\ln \left(2 / \theta_{0}\right)\left(1+\theta_{0}^{2}-\mu^{2}\right)},
$$

where $\mu=\cos \theta$ and $\theta_{0}$ is a small angle, which is estimated to be $\sim 0.01$ for winds from low-mass stars. The total momentum of the outflow, $p_{w}$, is calculated by assuming a fixed ratio of mass injected into the outflow relative to mass accretion to the star, $\dot{m}_{w} / \dot{m}_{*}=0.084$ in the fiducial model of Tan \& Blackman. The speed of the outflow is set equal to the escape speed from the stellar surface. As the star grows in mass and contracts to the main sequence the cumulative specific momentum of the outflow per stellar mass rises from $p_{w} / m_{*} \simeq 7 \mathrm{~km} \mathrm{~s}^{-1}$ when $m_{*} \simeq 10 M_{\odot}$ to $p_{w} / m_{*} \simeq 100 \mathrm{~km} \mathrm{~s}^{-1}$ when $m_{*} \simeq 100 M_{\odot}$.

To evaluate the efficiency of star formation from a core we find the angle, $\theta_{\text {esc }} \equiv \cos ^{-1} \mu_{\text {esc }}$, where the wind sweeps up core material to the surface escape speed of the core, $v_{\mathrm{esc}, \mathrm{c}}$. The location of the surface of Pop III.1 cores is not particularly well defined: the fiducial core of Tan \& McKee [26], based on the simulation results of Abel, Bryan, \& Norman [23], has about $1000 M_{\odot}$ of virialized baryonic material with a few $\times 10^{4} M_{\odot}$ of surrounding gas still infalling. These cores have $v_{\text {esc }, \mathrm{c}}=3.22\left(M / 1000 M_{\odot}\right)^{-1 / 7} K^{15 / 7} \mathrm{~km} / \mathrm{s}$. To find the speed of the swept-up gas, the analysis assumes thin, radiative shocks, purely radial motion and monopole gravity. We consider cores with angular mass distributions of the form $d M / d \Omega=(1 / 4 \pi) Q(\mu) M$ with $Q(\mu)=\left(1-\mu^{2}\right)^{n} / \int_{0}^{1}\left(1-\mu^{2}\right)^{n} d \mu$, and evaluate models with $n$, ranging from 0 , isotropic, to 4 , which describes a flattened distribution that mimics the effect of some rotational support. In the notation of Matzner \& McKee [50], the escape condition is given by $\left(1+\theta_{0}^{2}-\mu_{\mathrm{esc}}^{2}\right) Q\left(\mu_{\mathrm{esc}}\right)=m_{*} /(X M)$, where $X=$ $0.132 c_{g}\left[\ln \left(2 / \theta_{0}\right) / \ln 200\right] v_{\text {esc }, \mathrm{c}, 5}\left[\left(p_{w} / m_{*}\right) / 40 \mathrm{~km} \mathrm{~s}^{-1}\right]^{-1}$, and $c_{g}$ is a factor of order unity that accounts for the effects of gravity on shock propagation. We estimate $c_{g} \simeq 4.6$, for steady winds that decouple from the sweptup shell at the core edge in a core with $k_{\mathcal{O}}=2.2$ (which is the fiducial value of Tan \& McKee [26]). We ignore the influence of material beyond the core "edge", which is partly balanced by our neglect of the wind's influence on the shell beyond this point.

The star formation efficiency due to protostellar outflow winds is given by

$$
\varepsilon_{w}=\frac{1}{1+\left(\dot{m}_{w} / \dot{m}_{*}\right)\left(1-\phi_{w}\right)} \int_{0}^{\mu_{\mathrm{esc}}} Q \mathrm{~d} \mu,
$$

where $\phi_{w} \equiv \int_{0}^{\mu_{\mathrm{esc}}} P \mathrm{~d} \mu$ and $P(\mu)$ is the dimensionless force distribution in angle. Note that this definition of efficiency includes the effect of diversion of a fraction $\dot{m}_{w} / \dot{m}_{*}=0.084$ of the stellar accretion rate into outflow material. Equation 27 can be evaluated as a function of $m_{*}$ (see Fig. 11). $\theta_{\text {esc }}$ gradually increases and $\varepsilon_{w}$ decreases as the star grows and injects more momentum into its surroundings. However, note that $\varepsilon_{w}$ is an upper limit to the star formation efficiency if it is evaluated before the final stellar mass is reached since some material at $\theta>\theta_{\text {esc }}$ would later be ejected. Note also that the simple models shown here do not self-consistently account for the effects that a smaller mass accretion rate would have on protostellar evolution and outflow strength. Nevertheless they show that by the time $m_{*}$ has reached a hundred to a few hundred solar masses, the outflows have been able to eject about half the initial core material.

Figure 11 also shows the instantaneous accretion efficiency to the star + disk, $\varepsilon_{* d}$, due to radiative feedback and disk-shadowing for the fiducial $K^{\prime}=1, f_{\text {Kep }}=0.5$, $T_{i, 4}=2.5$ model. Comparison of these efficiencies is complicated by the fact that, as mentioned, for $\varepsilon_{w}$ at a given $m_{*}$, some gas at angles $>\theta_{\text {esc }}$ will eventually be 
ejected if the outflow continues. Nevertheless, we see that radiative feedback and disk-shadowing are more important, i.e. lead to smaller efficiencies, than mechanical feedback for masses $\gtrsim 50 M_{\odot}$ in the fiducial case. The outflow would need to operate until $m_{*} \sim 1000 M_{\odot}$ to reduce $\varepsilon_{w}$ to the value of $\varepsilon_{* d}$ seen at $m_{*} \simeq 100 M_{\odot}$. The possibility of lateral deflection of gas streamlines by the outflow is not treated by the above analysis, but we expect this would tend to increased $\varepsilon_{w}$. This effect can be studied with numerical simulations. Machida et al. [52] have carried out 3D MHD simulations of the very earliest stages of protostellar outflow feedback, up to times when the protostar has just formed with $m_{*} \lesssim 0.01 M_{\odot}$. Such simulations need to be advanced to later stages to explore the effects of a realistic 3D geometry on the outflow-core interaction and star formation efficiency.

While mechanical feedback is unlikely to dominate over radiative feedback, it may influence details of the radiative feedback processes. Even a small outflow cavity when $m_{*} \sim 20 M_{\odot}$ will help dissipate Ly- $\alpha$ photons and prevent a build up of dynamically important radiation pressure at this stage. An outflow will tend to facilitate $\mathrm{H}$ II region breakout in polar directions by lowering gas densities and injecting additional momentum. As it is launched from the surface of the disk, a dense bipolar outflow can confine the protostellar ionizing flux in equatorial directions and prevent it reaching outer disk to cause photoevaporation [53]. However, Tan \& Blackman [40] showed that the fiducial outflow would not be dense enough to significantly shield the disk from the ionizing luminosities of primordial protostars once $m_{*} \gtrsim 40 M_{\odot}$. On the other hand, the outflow is likely to impact the structure of the ionized disk atmosphere and thus affect the photoevaporation mass loss rate, perhaps similar to the "strong stellar wind" case of Hollenbach et al. [49].

\section{Impact of Pop III.1 Stars for Larger-Scale Feedback and Metal Production}

The predicted final protostellar masses (i.e. the initial stellar masses) for the range of model parameters we have considered are summarized in Table 1. The stars are all "massive" (i.e. $m_{*}>8 M_{\odot}$ ) and will thus have significant radiative and mechanical feedback on their surroundings, and the potential for substantial metal enrichment via core-collapse or pair-instability supernovae.

Ionization, outflow, wind and supernova feedback can lead to the disruption and unbinding of gas from nearby minihalos In particular ionization is likely to be the first and most important feedback effect to initiate unbinding: ionizing radiation heats up the gas to temperatures $~$ $25,000 \mathrm{~K}$ [16, 17], depending on the temperature of the radiation field, with corresponding sound speeds that

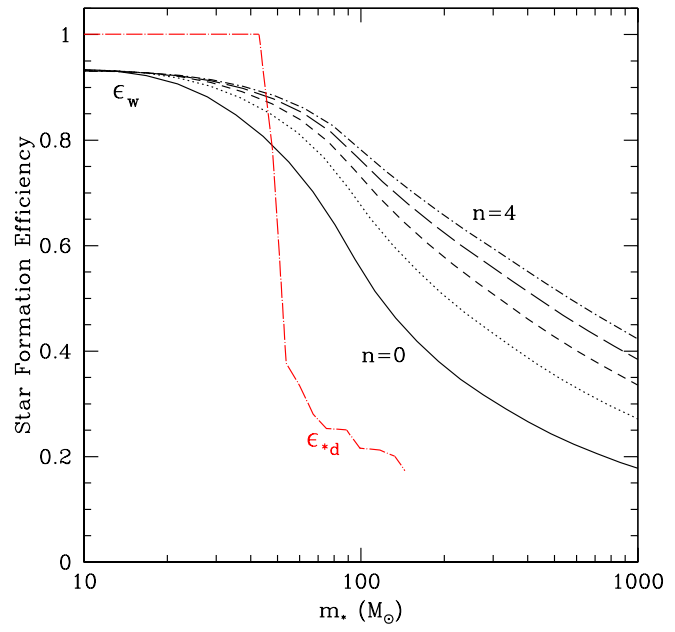

FIGURE 11. Evolution of star formation efficiency, $\varepsilon_{w}$, due to erosion of a $1000 M_{\odot}$ gas core by protostellar outflows winds for the fiducial $f_{\text {Kep }}=0.5$ and $K^{\prime}=1$ case, with $\alpha_{\text {ss }}=0.01$, and ignoring radiative feedback processes [40]. The density profile of the initial core is specified by $d M / d \Omega=(1 / 4 \pi) Q(\mu) M$, with $\mu=\cos \theta$ and $Q(\mu)=\left(1-\mu^{2}\right)^{n} / \int_{0}^{1}\left(1-\mu^{2}\right)^{n} d \mu$. Solid line is $n=0$ (isotropic core), dotted is $n=1$, dashed is $n=2$, long-dashed is $n=3$, dot-dashed is $n=4$. The dot-long-dashed line shows the instantaneous efficiency, $\varepsilon_{* d}$, due to radiative feedback processes (i.e. H II region breakout, limited by diskshadowing $)$ for the fiducial model $\left(f_{\mathrm{Kep}}=0.5, K^{\prime}=1, T_{i, 4}=\right.$ $2.5)$, indicating their greater importance at $m_{*} \gtrsim 50 M_{\odot}$.

are much larger than the few $\mathrm{km} / \mathrm{s}$ typical of minihalos. Note, however, that in addition to unbinding gas from minihalos, the pressure forces associated with ionized regions can shock-compress adjacent neutral regions and promote gravitational collapse, and this possibility is discussed in the next section. Stars with masses in the range shown in Table 1 are able to completely ionize at least their local minihalo, and more typically a much large volume of the surrounding intergalactic medium. For recent numerical simulations of photoevaporation of minihalos see Whalen et al. [54] and references therein.

Lyman-Werner band radiation destroys $\mathrm{H}_{2}$ molecules, reducing the cooling efficiency of the gas. We can estimate the distance over which star formation is suppressed from the work of Glover \& Brand [55]. Assuming that the core is in approximate hydrostatic equilibrium and is characterized by an entropy parameter $K$, we find [15] that the time to dissociate $\mathrm{H}_{2}$ is less than the free-fall time $t_{\mathrm{ff}}$ if the core is within a distance

$$
D=\left(\frac{S_{\mathrm{LW}}}{10^{49} \mathrm{~s}^{-1}} \frac{10^{-3}}{x_{2}} \frac{f_{\text {abs }} f_{\text {diss }}}{0.01}\right)^{1 / 2} \frac{24}{\bar{n}_{4}^{21 / 40} K^{11 / 4}} \mathrm{pc},
$$

of the protostar, where $S_{\mathrm{LW}}$ is the photon luminosity in the Lyman-Werner bands, $x_{2}$ is the fractional abundance 
of $\mathrm{H}_{2}, f_{\mathrm{abs}}$ is the fraction of the Lyman-Werner flux absorbed by the $\mathrm{H}_{2}, f_{\text {diss }}$ is the fraction of absorptions that result in dissociation, and $\bar{n}$ is the mean density of $\mathrm{H}$ nuclei. Thus, even a $100 M_{\odot}$ star, which has $S_{\mathrm{LW}} \simeq$ $10^{49} \mathrm{~s}^{-1}$, can suppress star formation in an existing core only if the core is relatively nearby. A more detailed analysis by Susa [56] comes to similar conclusions (see also Ahn \& Shapiro [57] and Whalen et al. [54]).

If gas ionized by the Pop III.1 star has a chance to recombine, e.g. once the star completes its nuclear burning, the relatively high residual electron fraction catalyzes molecule formation, particularly of HD, which can dramatically enhance subsequent cooling [20]. This is the most likely scenario for the next generation of star formation, Population III.2 (discussed in the next section).

Lacking metal lines in their atmospheres, mainsequence and post-main-sequence Pop III stars are expected to have quite weak stellar winds [58]. This also implies their mass at the end of stellar evolution should be similar to their initial mass. Rotation is expected to enhance mass loss [59], although the final mass is still thought to be a significant fraction of the initial mass.

Thus the range of final protostellar masses predicted by models of radiative and mechanical feedback (Table (1) imply a range of final pre-core-collapse stellar masses that potentially overlap the range of masses necessary to produce pair instability supernovae, $140-$ $260 M_{\odot}$ in the models of Heger \& Woosley [60]. Rotation may lower these limits (S. Woosley, private comm.). The lack of the expected nucleosynthetic signature of such supernovae in the abundance patterns of very metal poor halo stars [61], could indicate that such massive Pop III.1 stars were relatively rare or that they tended to enrich regions not probed by typical halo stars, perhaps the centers of larger galactic halos. The conclusion by Scannapieco et al. [62] that Pop III star formation should be fairly widespread in regions now probed by Galactic halo stars, can be reconciled with the abundance pattern observations if most of this star formation leads to either Pop III.1 stars from relatively low entropy $\left(K^{\prime} \lesssim 1\right)$ gas cores or Pop III. 2 stars (discussed below) that also have a mass scale below the pair instability threshold (see also the study by Greif \& Bromm [21]). Further work is required to determine the range of pre-stellar core parameters, primarily $K^{\prime}$ and $f_{\mathrm{Kep}}$, exhibited in cosmological simulations, in order to predict the frequency of pair instability supernovae.

\section{POPULATION III.2 STAR FORMATION}

Population III. 2 stars are those with near primordial composition, so that their formation and stellar evolution are independent of metallicity, but with initial conditions "significantly" affected by radiative and/or mechanical feedback from previous stellar generations (that could be Pop III.1, Pop III.2, or even Pop II or Pop I) or AGN.

We expect radiative feedback (especially FUV destruction of $\mathrm{H}_{2}$ ) to be far more pervasive than the direct mechanical feedback associated with protostellar outflows, winds or supernovae, although shocks driven by D-type ionization fronts will be even more penetrating than ionization into dense regions. Such shock compression may be important in promoting star formation in neighboring minihalos of intermediate central density, $10 \mathrm{~cm}^{-3} \lesssim n_{\mathrm{H}} \lesssim 1000 \mathrm{~cm}^{-3}$ [54], although the properties of the stars that subsequently form under these conditions remain to be determined. Stacy \& Bromm [63] have discussed the impact of cosmic rays produced from Pop III stars, which would also be highly penetrating, although estimates of their production rate are uncertain.

In gas that is no longer exposed to significant ionizing and dissociating flux and that has a chance to recombine, the relatively high residual electron fraction catalyzes molecule formation, particularly of HD, which can dramatically enhance subsequent cooling, perhaps reducing the characteristic star-formation mass[20, 21]. Such a reduction is in qualitative agreement with our models with lower values of $K^{\prime}$, which is proportional to the temperature (eq. 1): such stars reach the main sequence (and associated higher ionizing luminosities) at lower protostellar masses. They also have smaller rates of accretion that they need to disrupt via disk photoevaporation. The precise mass range that is applicable for typical Pop III.2 stars requires more detailed numerical and analytic study, but initial estimates, based on the $K^{\prime}=0.5$ model, suggest that their masses are significantly smaller than those necessary to produce pair instability supernovae [60].

\section{THE TRANSITION TO POPULATION II}

Metal atoms and dust grains provide additional mechanisms for the gas to cool. For a given temperature, density and ionization history there is some "critical metallicity" at which these will start to influence star formation by dominating over $\mathrm{H}_{2}$ and $\mathrm{HD}$ cooling.

The value of this critical metallicity for star formation is uncertain, with estimates ranging from $\sim 10^{-6} Z_{\odot}$ if the cooling is dominated by small dust grains that contain a significant fraction of the metals [18] to $\sim 10^{-3.5} Z_{\odot}$ if the cooling is dominated by the fine structure lines of $\mathrm{C}$ and $\mathrm{O}$ and there is negligible $\mathrm{H}_{2}$ [19]; if $\mathrm{H}_{2}$ cooling is included, Jappsen et al. [64] argue that there is no critical metallicity for gas-phase metals.

A detailed understanding of the transition to Pop II star formation will require knowledge of the Pop III.1 and III.2 IMFs, their mass loss during stellar evolution due to stellar winds and the metal and dust content of these winds, the metal and dust content of their super- 
nova ejecta, the mixing of wind and supernova ejected metals and dust into the surrounding (dense) gas that subsequently becomes gravitational unstable. It is fair to say that such a complicated problem, with so many uncertain inputs, remains far from solved. The general consensus is that, with additional coolants the gas can fragment into smaller pre-stellar cores that will tend to have smaller accretion rates. Both of these effects should lead to an IMF peaked at smaller masses than that of Pop III stars.

\section{COMPARISON TO PRESENT-DAY STAR AND STAR CLUSTER FORMATION}

\section{Initial Conditions}

The mean gas densities and velocity dispersions of Pop III.1 minihalos and present-day star-forming gas clumps [65] are quite similar. The major difference is that in present-day gas, cooling by molecules and dust proceeds down to $\sim 10 \mathrm{~K}$ so that thermal pressure becomes negligible compared to other sources, such as magnetic and turbulent pressure. A magnetized, supersonicallyturbulent, self-gravitating gas clump is prone to fragmentation: stochastic shock compressions produce a range of unstable core masses down to about the thermal Jeans mass. This process is likely to be responsible for shaping the present-day stellar IMF. Although Pop III.1 cores are permeated by mild (sonic) turbulence, the compressions produced by these fluctuations are quite weak, and, coupled with the weak cooling properties of primordial composition gas, means that fragmentation is suppressed. As a result, the IMF of Pop III.1 stars is likely to be qualitatively different from that of Pop II and Pop I stars: it should be set by the distribution of the entropy parameter $K^{\prime}$ and the rotation parameter $f_{\text {Kep }}$. Most numerical simulations of Pop III.1 star formation have not exhibited fragmentation in the minihalo down to their resolution limits, unless it occurs in a rotationally supported disk. Even this latter case appears to be unlikely to occur for realistic cosmological initial conditions.

A second difference is that the sites of Pop III.1 star formation are determined by dark matter potentials, i.e. the centers of minihalos. The cooling and settling of baryons mean that these typically dominate the mass densities on scales inside $\sim 1$ pc, but the dark matter still affects the gravitational potential of the larger scale core, which can affect the efficiency of feedback mechanisms. Dark matter annhilation in the centers of the halos could provide an important heating mechanism that could delay the onset of star formation [33].

\section{Feedback Mechanisms}

One may ask how the feedback mechanisms of Pop III.1 stars relate to those that operate in contemporary massive star formation. We note that the maximum mass attained in the fiducial model of McKee \& Tan of Pop III.1 star formation [15] is very similar to that inferred observationally in local massive star clusters (e.g. [66]). However, after decades of study, it remains unclear whether the maximum mass of stars forming today is set by feedback or instabilities in very massive stars [67]. We have argued that the maximum mass of primordial stars is set by feedback. The primary differences in the feedback processes then and now are:

(1) Dust. In contemporary star-forming regions, dust destroys Lyman- $\alpha$ photons, eliminating them as a significant pressure. On the other hand, the dust couples the pressure of the UV continuum radiation to the gas very effectively, and it remains to be determined whether this limits the final mass of the star; e.g., Yorke \& Sonnhalter [68] find that it does, whereas Krumholz et al. [69] have not found evidence that it does. Dust also affects the evolution of $\mathrm{H}$ II regions, absorbing a significant fraction of the ionizing photons in dense H II regions, thereby reducing the impact of $\mathrm{H}$ II region breakout and disk photoevaporation feedback.

(2) Magnetic fields. In contemporary protostars, magnetic fields drive powerful winds that drive away a significant fraction of the core out of which the star is forming [50]. The cavities created by these winds allow radiation to escape from the vicinity of the protostar, significantly reducing the radiation pressure [70]. As we have discussed, it is uncertain whether primordial protostars have such powerful magnetically-driven outflows [40]. However, even if they are present, we have argued that they would not be the dominant mechanism compared to disk photoevaporation in setting the final stellar mass.

(3) Stellar temperatures and luminosities. Primordial stars were significantly hotter than contemporary stars, resulting in significantly greater ionizing luminosities. In addition, the accretion rates of primordial massive stars are much greater, at least initially, than those of contemporary massive stars [71].

\section{ACKNOWLEDGMENTS}

We thank many colleagues for helpful discussions, including T. Abel, F. Adams, V. Bromm, A. Ferrara, K. Freese, G. Meynet, A. Natarajan, B. O'Shea, E. Scannapieco, D. Spolyar, D. Whalen, S. Woosley and N. Yoshida. The research of JCT is supported by NSF CAREER grant AST-0645412. The research of CFM is supported by NSF grants AST-0606831 and PHY05-51164. 


\section{REFERENCES}

1. L. Page, G. Hinshaw, E. Komatsu et al., ApJS, 170, 335-376 (2007).

2. M. F. Morales, and J. Hewitt, ApJ, 615, 7-18 (2004).

3. T. C. Beers, and N. Christlieb, ARA\&A, 43, 531-580 (2005).

4. J. Schaye, A. Aguirre, T-S. Kim et al., ApJ, 596, 768-796 (2003).

5. M. L. Norman, B. W. O'Shea, and P. Paschos, ApJ, 601, L115-L118 (2004).

6. M. R. Santos, V. Bromm, and M. Kamionkowski, MNRAS 336, 1082-1092 (2002).

7. E. R. Fernandez, and E. Komatsu, ApJ, 646, 703-718 (2006).

8. A. Kashlinsky, R. Arendt, J. P. Gardner, et al., ApJ, 608, $1-9$ (2004).

9. R. I. Thompson, D. Eisenstein, X. Fan, et al., Apj, 657, 669-680 (2007).

10. S. M. Weinmann, and S. J. Lilly, ApJ, 624, 526-531 (2005).

11. V. Bromm, and A. Loeb, ApJ, 575, 111-116 (2002).

12. D. P. Stark, R. S. Ellis, J. Richard, et al., ApJ, 663, 10-28 (2007).

13. X. Fan, M. A. Strauss, Schneider, D. P. et al., $A J, \mathbf{1 2 5}$, 1649-1659 (2003).

14. C. J. Willott, R. J. McLure, and M. J. Jarvis, ApJ, 587, L15-L18 (2003).

15. C. F. McKee, and J. C. Tan, Apj, submitted, astro$\mathrm{ph} / 0711.1377,(2008)$

16. M. L. Giroux, \& P. R. Shapiro, ApJS, 102, 191-238 (1996).

17. P. R. Shapiro, I. T. Iliev, \& A. C. Raga, MNRAS, 348, 753-782 (2004).

18. K. Omukai, T. Tsuribe, R. Schneider, and A. Ferrara, ApJ, 626, 627-643 (2005)

19. V. Bromm, and A. Loeb, Nature, 425, 812-814 (2003).

20. H. Uehara, and S-I. Inutsuka, ApJ, 531, L91-94 (2000).

21. T. H. Greif, and V. Bromm, MNRAS, 373, 128-138 (2006)

22. M. Tegmark, J. Silk, M. J. Rees, A. Blanchard, T. Abel, and F. Palla, ApJ, 474, 1-12 (1997).

23. T. Abel, G. L. Bryan, and M. L. Norman, Science, 295, 93-98 (2002).

24. V. Bromm, P. S. Coppi, and R. B. Larson, ApJ, 564, 23-51 (2002).

25. V. Bromm, and A. Loeb, New Ast., 5, 353-364 (2004).

26. J. C. Tan, and C. F. McKee, ApJ, 603, 383-400 (2004).

27. K. Omukai, and R. Nishi 1998, ApJ, 508, 141-150 (1998).

28. E. Ripamonti, F. Haardt, A. Ferrara, and M. Colpi, MNRAS, 334, 401-418 (2002).

29. C. Hunter, C. ApJ, 218, 834-845 (1977).

30. F. H. Shu, Apj, 214, 488-497 (1977).

31. N. Yoshida, K. Omukai, L. Hernquist, and T. Abel, Apj, 652, 6-25 (2006).

32. B. W. O'Shea, and M. L. Norman, Apj, 654, 66-92 (2007).

33. D. Spolyar, K. Freese, P. Gondolo, astro-ph/0705.0521 (2007).

34. S. W. Stahler, F. Palla, \& E. E. Salpeter, Apj, 302, 590-605 (1986).

35. K. Omukai, and F. Palla, Apj, 589, 677-687 (2003).

36. D. Schaerer, $A \& A, \mathbf{3 8 2}, 28-42$ (2002).

37. C. F. Gammie, Apj, 553, 174-183 (2001).
38. F. C. Adams, S. P. Ruden, \& F. H. Shu, Apj, 347, 959-976 (1989).

39. F. H. Shu, S. Tremaine, F. C. Adams, and S. P. Ruden, Apj, 358, 495-514 (1990).

40. J. C. Tan, and E. G. Blackman, Apj, 603, 401-413 (2004).

41. S. A. Balbus, and J. F. Hawley, Rev. Mod. Phys., 70, 1-53 (1998).

42. R. M. Kulsrud, R. Cen, J. P. Ostriker, \& D. Ryu, Apj, 480, 481-491 (1997).

43. E. G. Blackman, and G. B. Field, Phys. Rev. Lett., 89, 265007 (2002).

44. N. I. Shakura, and R. A. Sunyaev, A\&A, 24, 337-355 (1973).

45. J. Frank, A. King, \& D. Raine, Accretion Power in Astrophysics (Cambridge: Cambridge Univ. Press) (1995).

46. I. V. Artemova, G. S. Bisnovatyi-Kogan, G. Bjoernsson, \& I. D. Novikov, Apj, 456, 119-123 (1996).

47. F. H. Shu, The Physics of Astrophysics Vol II: Gas Dynamics (Mill Valley: University Science Books) (1992).

48. C. A. Iglesias, and F. J. Rogers, Apj, 464, 943-953 (1996)

49. D. Hollenbach, D. Johnstone, S. Lizano, F. Shu, Apj, 428, 654-669 (1994).

50. C. D. Matzner, and C. F. McKee, Apj, 545, 364-378 (2000).

51. C. D. Matzner, and C. F. McKee, Apj, 526, L109-L112 (1999).

52. M. N. Machida, K. Omukai, T. Matsumoto, S-I. Inutsuka, Apj, 647, L1-L4 (2006).

53. J. C. Tan, and C. F. McKee, in IAU Symp. 221, Star Formation at High Angular Resolution, ed. M. Burton, R. Jayawardhana, \& T. Bourke (San Francisco: ASP), astro-ph/0309139 (2004).

54. D. Whalen, B. W. O'Shea, J. Smidt, M. L. Norman, Apj, submitted, astro-ph/0708.1603 (2008).

55. S. C. O. Glover, and P. W. J. L. Brand, MNRAS, 321, 385-397 (2001).

56. H. Susa, Apj, 659, 908-917 (2007).

57. K. Ahn, and P. R. Shapiro, MNRAS, 375, 881-908 (2007).

58. R. Kudritzki, Apj, 577, 389-408 (2002).

59. G. Meynet, S. Ekström, A. Maeder, A\&A 447, 623-639 (2006).

60. A. Heger, and S. E. Woosley, Apj, 567, 532-543 (2002).

61. J. Tumlinson, A. Venkatesan, and J. M. Shull, Apj, 612, 602-614 (2004).

62. E. Scannapieco, D. Kawata, C. B. Brook, R. Schneider, A. Ferrara, B. K. Gibson, Apj, 653, 285-299 (2006).

63. A. Stacy, and V. Bromm, MNRAS, in press, astro$\mathrm{ph} / 0705.3634,(2007)$.

64. A. K. Jappsen, R. S. Klessen, S. C. O. Glover, \& M.-M. Mac Low, astro-ph/0709.3530 (2007).

65. K. E. Mueller, Y. L. Shirley, N. J. Evans, \& H. R. Jacobson, ApJS, 143, 469-497 (2002).

66. D. F. Figer, Nature, 434, 192-194 (2005).

67. R. B. Larson, and S. Starrfield, A\&A, 13, 190-197 (1971).

68. H. W. Yorke, and C. Sonnhalter, Apj, 569, 846-862 (2002).

69. M. R. Krumholz, C. F. McKee, R. I. Klein, in Massive Star Birth: A Crossroads of Astrophysics, ed. R. Cesaroni, M. Felli, E. Churchwell, \& M. Walmsley (Cambridge: Cambridge University Press), p. 231 (2005).

70. M. R. Krumholz, C. F. McKee, R. I. Klein, Apj, 618, L33-36 (2005)

71. C. F. McKee, and J. C. Tan, Apj, 585, 850-871 (2003). 